\title{
Personality Characteristics of Patients with "White Coat" Hypertension
}

\author{
Shinjiro Muneta, Takamasa Kobayashi, and Isao Matsumoto
}

\begin{abstract}
To clarify psychological factors related to white coat hypertension, we examined personality characteristics of patients with mild essential hypertension by psychological testing. Patients with essential hypertension were taught to measure their own blood pressure (BP) with a semi-automatic oscillometric BP measuring device and were asked to measure BP at home in the sitting position before going to sleep. The duration of the study was $8 \mathrm{wk}$. Patients were defined as "white coat" hypertensive patients (WCHT) $(n=49)$ if home systolic BP was $135 \mathrm{mmHg}$ or less and home diastolic BP was $85 \mathrm{mmHg}$ or less, and as "sustained" hypertensive patients (SHT) $(n=53)$ if home systolic BP was $140 \mathrm{mmHg}$ or more or home diastolic BP was $90 \mathrm{mmHg}$ or more. All the patients underwent the following psychometric tests: self-rating questionnaire for depression, MMPI alexithymia scale, type A behavior pattern check list, general health questionnaire (GHQ), and egogram check list. WCHT did not differ from SHT in the scores for depression, alexithymia, type A behavior pattern, or GHQ. However, WCHT showed an abnormal pattern on egograms, as compared with SHT. On egograms, SHT showed a normal hill-shaped pattern with a peak in "nurturing parent (NP)", and "free child (FC)" was higher than "adapted child (AC)" in both genders. In contrast, WCHT showed significantly lower FC and significantly higher AC than SHT, and AC was higher than FC in both genders. These findings suggested that WCHT tend to suppress their own emotions and become over-adaptive to their surroundings, as compared with SHT. (Hypertens Res 1997; 20: 99-104)
\end{abstract}

Key Words: white coat hypertension, sustained hypertension, egogram, alexithymia, type A behavior pattern

It is well known that blood pressure (BP) readings measured by physicians at their offices are higher than those recorded by patients at home. The pressor response produced by the physician or other medical personnel in a clinical setting is called "white coat" phenomenon. Mancia et al. $(1,2)$ have shown that a physician's visit induced a substantial and immediate rise of BP in hospitalized patients with the use of continuous intra-arterial BP recording. The term "white coat" hypertension has been used to describe patients whose BP is elevated in the clinic but normal at home or on ambulatory BP monitoring. This occurs in as many as about $20 \%$ of patients with mild hypertension $(3,4)$. Patients with white coat hypertension may have a greater tendency to experience negative emotions such as anxiety, depression, and hostility than do patients whose BP is elevated both in the clinic and on ambulatory monitoring ("sustained" hypertension). However, many studies have failed to identify psychological characteristics that differentiate white coat hypertensive patients from sustained hypertensive patients $(5,6)$. Furthermore, these two types of patients do not differ with respect to pressor responses either to physical or mental stressors (5-7).
Psychophysiological factors contributing to white coat hypertension are still unclear.

To identify differences in psychological characteristics between patients with white coat hypertension and those with sustained hypertension, we performed psychometric tests and assessed the intensity of depression, alexithymia, neurosis, type A behavior pattern, and ego states in patients with mild essential hypertension.

\section{Subjects and Methods}

\section{Subjects}

The studies were carried out in essential hypertensive patients who had been followed up at our hospital. Essential hypertension was diagnosed when the systolic BP was $140 \mathrm{mmHg}$ or more or the diastolic BP was $90 \mathrm{mmHg}$ or more in the outpatient clinic on at least three separate visits over three months. BP was measured with the patient in the sitting position after a five minutes' rest. A standard mercury sphygmomanometer (phase $\mathrm{V}$ diastolic) was used for all measurements. Secondary forms of hypertension were ruled out by routine physical and laboratory examinations. None of the patients re-

From the Department of Internal Medicine, Matsuyama Red Cross Hospital, Matsuyama, Japan.

Address for Reprints: Shinjiro Muneta, M.D., Department of Internal Medicine, Matsuyama Red Cross Hospital, Bunkyo-cho 1, Matsuyama, Ehime 790, Japan.

Received October 9, 1996; accepted in revised form December 26, 1996. 
ceived any pharmacological treatment or were suffering from diseases that might have influenced their BPs. All patients had stage I or II hypertension according to the World Health Organization (WHO) stage classification. The patients were informed that they had high BP at the initial visit and were informed as to their severity of hypertension and extent of organ damage before enrollment in this study.

\section{Home and Clinic BP Measurements}

Home BP measurements were made with semiautomatic oscillometric BP measuring devices (HEM-709, 711, OMRON Co. Ltd, Tokyo, Japan) $(8,9)$. Patients were taught how to take their own BP correctly and were given a data sheet to record home BP readings at "hypertension education classes" held monthly at our hospital. In the present study, home BP was measured in the non-dominant arm using a standard arm cuff after a five minutes' rest in the sitting position before going to sleep. The home BP was measured on at least three separate days per wk for at least two months. The average of all home BP readings (at least 24 readings for each patient) was defined as the patient's home BP.

Clinic BP was measured in the non-dominant arm by the same male physician in the same examination room. A standard mercury sphygmomanometer was used for all measurements. The clinic BP was measured between 9:00 AM and 11:00 AM on three consecutive visits at 4-wk intervals during the same period that the home BP was measured. The average of the three readings was defined as the patient's clinic BP.

The accuracy of the semi-automatic BP measuring device was tested before the study by simultaneous auscultatory BP measurements in the dominant arm by a physician in the outpatient clinic at least three times. The differences between the average BP readings by the auscultatory method and those obtained with a BP measuring device were 10 $\mathrm{mmHg}$ or less for systolic BP and $5 \mathrm{mmHg}$ or less for diastolic BP. The BP measuring devices were not used if the differences in these two readings exceeded these values.

\section{Study Groups}

Essential hypertensive patients were defined as white coat hypertensive patients (WCHT) $(n=49$; 15 men and 34 women) if home systolic BP was 135 $\mathrm{mmHg}$ or less and home diastolic BP was $85 \mathrm{mmHg}$ or less, and as sustained hypertensive patients (SHT) ( $n=53 ; 21$ men and 32 women) if home systolic BP was $140 \mathrm{mmHg}$ or more or home diastolic BP was $90 \mathrm{mmHg}$ or more. Patients who could not be classified into these groups were excluded from analyses.

\section{Psychometric Tests}

All the patients underwent the following psychometric tests:

\section{1) Self-Rating Questionnaire for Depression (SRQ- D)}

SRQ-D is a screening test for masked depression, developed by Toho University (10). It consists of 18 items with 4 choices containing 6 control questions. A score of 10 or less is normal, a score from 11 to 15 is borderline, and a score of 16 or more is indicative of masked depression.

\section{2) Minnesota Multiphasic Personality Inventory (MMPI) Alexithymia Scale}

The MMPI alexithymia scale consists of 22 binary choice (true, false) questions (11). A score of 12 or less is normal, a score from 13 to 16 is borderline, and a score of 17 or more is indicative of alexithymia.

\section{3) Type A Behavior Pattern Questionnaire}

Type A behavior pattern was assessed by a triple choice (true, false, or neither) questionnaire designed by Maeda (12). The questionnaire consists of 12 items. A score of 17 or more is indicative of type A behavior pattern.

\section{4) General Health Questionnaire (GHQ)}

GHQ was developed by Goldberg in England (13) and was translated into Japanese by Nakagawa. It consists of 60 questions with 4 choices dealing with recent symptoms (14). The GHQ can effectively discriminate psychiatric patients from normal controls. Four choices were scored by the GHQ scoring method (from the left, $0-0-1-1$ ). The results were assessed on the basis of total GHQ score and scores for four factors (somatic symptoms, anxiety and sleep disturbance, social dysfunction, severe depression; 7 items for each factor). A total score of 17 or more is indicative of a neurotic tendency.

\section{5) Egogram Check List (ECL)}

In the theory of transactional analysis, human psychological energy is divided into five ego states: "critical parent (CP)", "nurturing parent (NP)", "adult (A)", "free child (FC)", and "adapted child (AC)" (15). The ECL is an egogram questionnaire developed by Sugita et al. $(16,17)$. It consists of 48 questions ( 8 items for each ego state and a lie scale) with triple choices (true, false, or neither). Scores of each ego scale can range from 0 to 8 .

\section{Statistical Analysis}

Group mean values of $\mathrm{BP}$ and age were compared by Student's $t$-test in each gender population. For scores of psychological tests, SHT and WCHT were compared by Mann-Whitney $U$-test in each gender population. The significance of differences in percentages between groups was evaluated by chisquare test. The level of significance was taken at $p$ $<0.05$. All data are expressed as the mean \pm SD.

\section{Results}

Table 1 shows the clinical characteristics of the patients. Most of the patients $(93.1 \%)$ were classified as having mild hypertension according to the guide- 
Table 1. Clinical Characteristics of Patients with Sustained Hypertension and White Coat Hypertension

\begin{tabular}{|c|c|c|c|c|}
\hline \multirow{2}{*}{ Variables } & \multicolumn{2}{|c|}{ Men } & \multicolumn{2}{|c|}{ Women } \\
\hline & SHT $(n=21)$ & WCHT $(n=14)$ & SHT $(n=32)$ & WCHT $(n=34)$ \\
\hline Age (yr) & $\begin{array}{c}59.5 \pm 13.0 \\
(34-85)\end{array}$ & $\begin{array}{c}60.1 \pm 11.5 \\
(38-80)\end{array}$ & $\begin{array}{c}58.6 \pm 9.7 \\
(40-75)\end{array}$ & $\begin{array}{c}59.2 \pm 9.5 \\
(38-75)\end{array}$ \\
\hline WHO II (\%) & 15.6 & 20.6 & 47.6 & 50.0 \\
\hline Clinic SBP (mmHg) & $157.4 \pm 10.5$ & $168.0 \pm 11.0$ & $156.1 \pm 13.8$ & $161.8 \pm 13.0$ \\
\hline Clinic DBP (mmHg) & $95.5 \pm 7.3$ & $90.5 \pm 11.1$ & $89.9 \pm 16.3$ & $92.3 \pm 6.2$ \\
\hline Home SBP (mmHg) & $146.8 \pm 9.6$ & $129.1 \pm 5.2$ & $143.4 \pm 7.5$ & $128.7 \pm 7.0$ \\
\hline Home DBP (mmHg) & $90.0 \pm 9.1$ & $76.4 \pm 6.9$ & $88.9 \pm 5.7$ & $77.1 \pm 6.2$ \\
\hline$\Delta$ Clinic-Home SBP $(\mathrm{mmHg})$ & $10.6 \pm 8.7$ & $38.9 \pm 10.4$ & $12.7 \pm 12.2$ & $33.1 \pm 12.5$ \\
\hline$\Delta$ Clinic-Home DBP $(\mathrm{mmHg})$ & $6.6 \pm 6.4$ & $14.0 \pm 9.3$ & $1.0 \pm 17.6$ & $15.2 \pm 6.7$ \\
\hline
\end{tabular}

SHT, sustained hypertension; WCHT, white coat hypertension; SBP, systolic blood pressure; DBP, diastolic blood pressure; WHO II, stage II according to the WHO stage classification; $\Delta$ Clinic-Home, difference between clinic and home blood pressures. Each value indicates the mean $\pm \mathrm{SD}$.

Table 2. Scores on the Self-Rating Questionnaire for Depression, the MMPI Alexithymia Scale, and the Questionnaire for Type A Behavior Pattern in Patients with Sustained Hypertension and White Coat Hypertension

\begin{tabular}{llcccccc}
\hline & & \multicolumn{2}{c}{ Men } & & \multicolumn{2}{c}{ Women } \\
\cline { 3 - 4 } & & SHT $(n=21)$ & WCHT $(n=14)$ & & SHT $(n=32)$ & WCHT $(n=34)$ \\
\hline SRQ-D & score $\geq 16(\%)$ & $7.3 \pm 4.1(4.8)$ & & $5.9 \pm 3.3(0.0)$ & & $6.8 \pm 9.7(3.1)$ & $7.0 \pm 4.1(2.9)$ \\
Alexithymia & score $\geq 17(\%)$ & $13.7 \pm 1.8(4.8)$ & $12.9 \pm 4.6(14.3)$ & & $15.8 \pm 2.3(31.3)$ & $15.2 \pm 1.9(26.5)$ \\
Type A & score $\geq 17(\%)$ & $14.7 \pm 6.4(42.9)$ & $10.4 \pm 2.8 *(0.0 * *)$ & & $13.0 \pm 5.3(28.2)$ & $13.9 \pm 4.9(32.4)$ \\
\hline
\end{tabular}

SHT, sustained hypertension; WCHT, white coat hypertension; SRQ-D, Self-Rating Questionnaire for Depression; Alexithymia, MMPI Alexithymia Scale; Type A, Type A Behavior Pattern Questionnaire. Each value indicates the mean \pm SD. ${ }^{*} p<0.05,{ }^{* *} p<0.01 v s$. SHT of the same gender.

Table 3. Total Scores and Scores of Four Factors on General Health Questionnaire in Patients with Sustained Hypertension and White Coat Hypertension

\begin{tabular}{|c|c|c|c|c|}
\hline & \multicolumn{2}{|c|}{ Men } & \multicolumn{2}{|c|}{ Women } \\
\hline & SHT $(n=21)$ & WCHT $(n=14)$ & SHT $(n=32)$ & WCHT $(n=34)$ \\
\hline Total score $\quad$ score $\geq 17(\%)$ & $11.0 \pm 6.3(23.8)$ & $8.1 \pm 4.7(7.1)$ & $12.6 \pm 8.6(25.0)$ & $10.7 \pm 9.1(23.5)$ \\
\hline \multicolumn{5}{|l|}{ Four factors } \\
\hline 1. Somatic symptoms & $2.4 \pm 1.7$ & $1.4 \pm 1.5$ & $2.4 \pm 2.2$ & $2.8 \pm 2.3$ \\
\hline $\begin{array}{l}\text { 2. Anxiety and sleep } \\
\text { disturbance }\end{array}$ & $2.0 \pm 1.9$ & $1.2 \pm 1.5$ & $1.9 \pm 1.9$ & $1.6 \pm 1.7$ \\
\hline 3. Social dysfunction & $0.8 \pm 0.9$ & $0.5 \pm 0.8$ & $0.7 \pm 0.9$ & $0.4 \pm 0.8$ \\
\hline 4. Severe depression & $0.4 \pm 0.9$ & $0.4 \pm 1.4$ & $0.4 \pm 0.8$ & $0.4 \pm 1.0$ \\
\hline
\end{tabular}

SHT, sustained hypertension; WCHT, white coat hypertension. Each value indicates the mean \pm SD.

lines from a WHO/ISH (International Society of Hypertension) meeting. There were no significant differences in age, clinic systolic BP, or clinic diastolic BP between SHT and WCHT in either gender. The percentage of patients with stage II hypertension according to the WHO stage classification (WHO II) in SHT was similar to that in WCHT in both genders. However, the percentage of patients with a diagnosis of WHO II was higher in women than in men, probably because the duration of hypertension was slightly longer in women than in men in the present study. In most of the patients with a diagnosis of WHO II, target organ damage was mild left ventricular hypertrophy on electrocardiogram and there were no other signs of organ involvement.

Table 2 shows the scores on the SRQ-D, MMPI alexithymia scale, and type A behavior pattern questionnaire of the patients. There were no significant differences in the SRQ-D scores between SHT and WCHT in either gender. Only three patients had a SRQ-D score of 16 or more. As for the MMPI alexithymia scale, women had slightly higher scores than men, but there were no significant dif- 
Table 4. Percentage of Patients Who Showed a Pattern of AC $>$ FC in Patients with Sustained Hypertension and White Coat Hypertension of Different Age Groups

\begin{tabular}{|c|c|c|c|c|}
\hline \multirow[b]{2}{*}{$(\%)$} & \multicolumn{2}{|c|}{ Men } & \multicolumn{2}{|c|}{ Women } \\
\hline & $\operatorname{SHT}(n=21)$ & WCHT $(n=14)$ & $\operatorname{SHT}(n=32)$ & WCHT $(n=34)$ \\
\hline Total & $23.8(5 / 21)$ & $85.7^{* *}(12 / 14)$ & $25.0(8 / 32)$ & $67.6^{* *}(23 / 34)$ \\
\hline Middle-age (age $<65 \mathrm{yr}$ ) & $21.4(3 / 14)$ & $87.5^{* *}(7 / 8)$ & $21.7(5 / 23)$ & $60.0^{* *}(15 / 25)$ \\
\hline Old-age (age $\geq 65$ yr) & $28.6(2 / 7)$ & $83.3 *(5 / 6)$ & $33.3(3 / 9)$ & $88.7^{*}(8 / 9)$ \\
\hline
\end{tabular}

SHT, sustained hypertension; WCHT, white coat hypertension. ${ }^{*} p<0.05,{ }^{* *} p<0.01 v s$. SHT of the same gender.
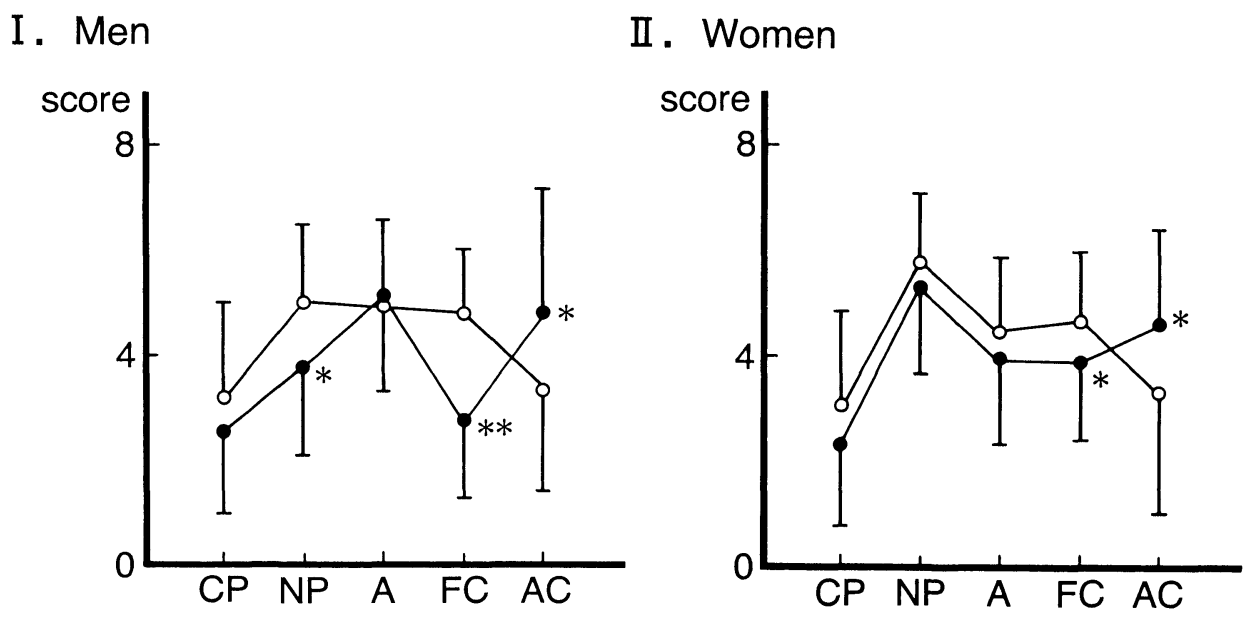

Fig. 1. Egogram patterns in patients with sustained hypertension and white coat hypertension. $\bigcirc:$ sustained hypertension, - : white coat hypertension, CP: critical parent, NP: nurturing parent, A: adult, FC: free child, AC: adapted child. Each point indicates the mean $\pm S D$. ${ }^{*} \mathrm{p}<0.05,{ }^{* *} \mathrm{p}<0.01$ vs. sustained hypertension.

ferences between SHT and WCHT in the scores or the percentage of patients who showed a score of 17 or more in either gender. As for the type A behavior pattern questionnaire, SHT had significantly $(p<0.05)$ higher scores and a significantly $(p<0.01)$ higher percentage of patients who showed a score of 17 or more than did WCHT in men, but there were no significant differences between SHT and WCHT in women.

Table 3 shows the total scores of GHQ and the scores of four factors by GHQ. No statistically significant differences were found between SHT and WCHT in either gender. SHT had a slightly, but not significantly, higher percentage of patients with a total score of 17 or more, as compared with WCHT in men.

Figure 1 shows the egogram patterns of SHT and WCHT. SHT showed a normal hill-shaped pattern with a peak in NP, and FC was higher than $\mathrm{AC}$ in both genders. In contrast, WCHT showed significantly ( $p<0.01$ for men, $p<0.05$ for women) lower FC and significantly $(p<0.05)$ higher AC than SHT, and $\mathrm{AC}$ was higher than FC in both genders. In addition to these findings, WCHT showed significantly $(p<0.05)$ lower NP than SHT in men.

Table 4 shows the percentage of patients who showed a pattern of AC $>$ FC in SHT and WCHT. The patients were divided into two age groups: middle-age (age $<65 \mathrm{yr}$ ) and old-age (age $\geq 65 \mathrm{yr}$ ).
The percentage of AC $>$ FC in WCHT was significantly higher than that in SHT in both genders in each age group. The $\mathrm{AC}>\mathrm{FC}$ pattern was seen in $72.9 \%(35 / 48)$ of all WCHT and in $24.5 \%(13 / 53)$ of all SHT.

\section{Discussion}

Many studies have sought to determine psychological factors related to white coat hypertension. Gerardi et al. (18) have reported that hypertensive patients with similar clinic and home BPs (nonresponders) showed a significantly higher extent of anger and hostility than did hypertensive patients with lower home BPs (office responders). Schneider et al. (19) reported that borderline hypertensive patients with high home BP showed a greater intensity of anger than did those with normal home BP. Lerman et al. (20) also found that white-coat responders reported less anger and less overt expression of anger than did nonresponders. All three of these studies showed similar differences in anger and hostility between WCHT and SHT. However, WCHT did not differ from SHT with respect to the intensity of anxiety and depression in many reports (5, 18-21). In the present study, we investigated the extent of masked depression, alexithymia, type A behavior pattern, and neurotic tendency in the two groups. WCHT did not differ from SHT in any of 
these psychological factors, except that SHT showed a significantly stronger tendency for type A behavior pattern than did WCHT in the case of men. One possible explanation for this finding is that male patients with type A behavior pattern may maintain high BP outside the clinic because of increased pressor reactivity to stress in their natural environment.

The theory of transactional analysis developed by Berne has been used in various fields, such as psychology, psychiatry, social work, education, religion, and business, since the mid 1960s (22). According to this theory, the structure of each individual's personality is based on ego states (23). Dusay developed the concept of the egogram, which graphically depicts five ego states (CP, NP, $\mathrm{A}, \mathrm{FC}, \mathrm{AC})$ in relation to one another (15). Although characteristic egogram patterns have been reported in patients with psychosomatic diseases, neurosis, depression, and certain mental disorders, there have been no studies of egogram patterns in WCHT. In the present study, we found that WCHT had low FC and high AC as compared with SHT, and AC was higher than FC in WCHT. The egogram pattern indicated that WCHT tend to suppress their own emotions and adapt themselves to others or to their surroundings, as compared with SHT. The male WCHT also showed low NP, suggesting that they tend to be uncommunicative. The "AC FC" pattern and low NP are often seen in patients with psychosomatic diseases, neurosis, and depression (24). These findings indicate that the transactional process of interpersonal interaction between doctor and patient may play an important role in the white coat phenomenon, and psychotherapy utilizing a transactional analysis approach may be useful in the management of white coat hypertension.

Several studies have reported that white coat hypertension is more frequent and white coat phenomenon is more prominent in women than in men with mild hypertension (25-28). Pickering et al. (3) suggested that this gender difference might be related to the stereotype of the physician as a male authority figure. In a study performed by McCubbin et al. (29), the effect of the screener's gender on resting BP values were evaluated by repeated measurements in an on-campus screening of college students. They found that the difference between initial and subsequent BP values was greater when measured by a screener of the opposite gender than when measured by a screener of the same gender. These findings suggest that white coat phenomenon may be in part attributable to the gender discordance between the screener and the subject, and thus psychosocial factors related to white coat hypertension might differ between genders. Furthemore, factors causing white coat hypertension may differ between young and elderly patients. However, in the present study, the egogram pattern of WCHT was similar in both genders, and in both middle-age and elderly patients. These findings suggest that WCHT have a common personality structure irrespective of gender and age.
Psychological factors have been shown to cause $\mathrm{BP}$ variation in young subjects. Rostrup et al. (30) showed that in 29 young patients psychological stress caused by awareness of hypertension or hypertension labeling may increase BP and sympathetic activity. Amigo et al. (31) have also reported that oral instructions about BP at the first measurement can influence BP at the next measurement in both normotensive and hypertensive subjects. Williams et al. (32) have noted the importance of interpersonal interaction as a determinant of cardiovascular responses to emotional stimuli. These studies suggest that hypertension should be diagnosed after repeated BP measurements and the establishment of a doctor-patient relationship. Before enrollment in this study, all patients were informed of their BP readings on each clinical visit at an interval of 2 or $4 \mathrm{wk}$ for at least 3 months, and were educated with regard to hypertension to eliminate possible psychological sources of variation in the measurement of BP.

White coat hypertension is defined as a persistently elevated clinic BP together with a normal daytime ambulatory pressure or normal pressure at home. However, the definition of elevated and normal in this context is quite arbitrary, and little information is available on the interpretation of selfmeasured home BP. Imai et al. (33) evaluated the distribution of home BP in untreated subjects in the general community in northern Japan, and reported that mean $+2 \mathrm{SD}$ and the 95 th percentile values of home BP in normotensive subjects were 137/86 and $134 / 83 \mathrm{mmHg}$, respectively. Other investigators have also reported that the upper limit of normal home BP (mean + 2SD) was around 135-140/85-90 $\mathrm{mmHg}(34-36)$. Therefore, in the present study we defined WCHT as hypertensive patients whose home systolic BP was 135 or less and home diastolic $\mathrm{BP}$ was $85 \mathrm{mmHg}$ or less.

In conclusion, WCHT did not differ from SHT in the extent of depression, alexithymia, type A behavior pattern, or neurotic tendency. However, ego state analysis indicated that WCHT tend to suppress their own emotions and adapt themselves to the surroundings to a greater extent than do SHT. Psychotherapy based on transactional analysis may be useful in the management of white coat hypertension.

\section{References}

1. Mancia G, Bertinieri G, Grassi G, et al: Effects of blood pressure measurement by the doctor on patient's blood pressure and heart rate. Lancet 1983; 2: 695-697.

2. Mancia G, Parati G, Pomidossi G, Grassi G, Casadei $\mathrm{R}$, Zanchetti A: Alerting reaction and rise in blood pressure during measurement by physician and nurse. Hypertension 1987; 9: 209-215.

3. Pickering TG, James GD, Boddie C, Harshfield GA, Blank S, Laragh JH: How common is white coat hypertension? JAMA 1988; 259: 225-228.

4. Pickering TG: Clinical applications of ambulatory blood pressure monitoring: the white coat syndrome. 
Clin Invest Med 1991; 14: 212-217.

5. Siegel WC, Blumenthal JA, Divine GW: Physiological, psychological, and behavioral factors and white coat hypertension. Hypertension 1990; 16: 140-146.

6. Julius S, Jamerson K, Gudbrandsson T, Schork N: White coat hypertension: a follow-up. Clin Exp Hypertens [A] 1992; 14: 45-53.

7. Cardillo C, De Felice F, Campia U, Folli G: Psychophysiological reactivity and cardiac end-organ changes in white coat hypertension. Hypertension 1993; 21: 836-844.

8. Foster C, McKinlay S, Cruickshank JM, Coats AJS: Accuracy of the Omron HEM 706 portable monitor for home measurement of blood pressure. J Hum Hypertens 1994; 8: 661-664.

9. O'Brien E, Mee F, Atkins N, Thomas M: Evaluation of three devices for self-measurement of blood pressure according to the revised British Hypertension Society Protocol: the Omron HEM-705CP, Philips HP5332, and Nissei DS-175. Blood Pressure Monitoring 1996; 1: 55-61.

10. Abe T, Tsutsui $\mathrm{S}$, Namba $\mathrm{T}$, et al: Studies on the self-rating questionnaire for the screening test of masked depression (SRQ-D). Seishin-Igaku 1972; 12: 243-247 (in Japanese).

11. Kleiger JH, Kinsman RA: The development of an MMPI alexithymia scale. Psychother Psychosom 1980; 34: 17-24.

12. Maeda S: A study on behavior pattern of patients with coronary heart diseases: application of brief questionnaire. Shinshin-Igaku 1985; 25: 297-306 (in Japanese).

13. Goldberg DP, Blackwell B: Psychiatric illness in general practice: a detailed study using a new method of case identification. $B r$ Med J 1970; 2: 439-443.

14. Fukunishi I, Hosokawa K, Nakagawa T: Psychosomatic health investigations on Tanshin-Funin in Kagawa. Shinsin-Igaku 1987; 27: 619-627 (in Japanese).

15. Dusay JM: Egograms: how I see you and you see me. Harper \& Row, New York, 1977.

16. Sugita M, Shinzato R, Wada M, Segawa K, Ishikawa $\mathrm{H}$ : On a new egogram check list (ECL). Koryubunseki-Kenkyu 1979; 4: 28-40 (in Japanese).

17. Ikemi Y, Sugita M: The oriental version of transactional analysis. Psychosomatics 1975; 16: 164-170.

18. Gerardi RJ, Blanchard EB, Andrasik F: Psychological dimensions of 'office hypertension'. Behav Res Ther 1985; 23: 609-612.

19. Schneider RH, Egan BM, Johnson EH, Drobny H, Julius S: Anger and anxiety in borderline hypertension. Psychosom Med 1986; 48: 242-248.

20. Lerman CE, Brody DS, Hui T, Lazaro C, Smith DG, Blum MJ: The white-coat hypertension response: prevalence and predictors. J Gen Intern Med 1989; 4: 226-231.

21. Laughlin KD, Sherrard DJ, Fisher L: Comparison of clinic and home blood pressure levels in essential hypertension and variables associated with clinichome differences. J Chronic Dis 1980; 33: 197-206.

22. Heyer NR: Development of a questionnaire to measure ego states with some application to social and comparative psychiatry. Trans Anal J 1979; 9: 9-19.

23. Hohmuth AV, Gormly AV: Ego state models and personality structure. Trans Anal J 1982; 12: 140143.

24. Sugita M: Koryu-Bunseki. Nihonbunka-Kagakusha, Tokyo, 1985, pp11-51 (in Japanese).

25. Høegholm A, Kristensen KS, Madsen NH, Svendsen TL: White coat hypertension diagnosed by 24-h ambulatory monitoring: examination of 159 newly diagnosed hypertensive patients. Am J Hypertens 1992; 5: 64-70.

26. Khoury S, Yarows SA, O'Brien TK, Sowers JR: Ambulatory blood pressure monitoring in a nonacademic setting: effects of age and sex. Am J Hypertens 1992; 5: 616-623.

27. Staessen JA, O'Brien ET, Atkins N, et al: Short report: ambulatory blood pressure in normotensive compared with hypertensive subjects. J Hypertens 1993; 11: 1289-1297.

28. Millar JA, Isles CG, Lever AF: Blood pressure, 'white-coat' pressor responses and cardiovascular risk in placebo-group patients of the MRC mild hypertension trial. J Hypertens 1995; 13: 175-183.

29. McCubbin JA, Wilson JF, Bruehl S, Brady M, Clark K, Kort E: Gender effects on blood pressures obtained during an on-campus screening. Psychosom Med 1991; 53: 90-100.

30. Rostrup M, Kjeldsen SE, Eide IK: Awareness of hypertension increases blood pressure and sympathetic responses to cold pressor test. Am J Hypertens 1990; 3: 912-917.

31. Amigo I, Cuesta V, Fernández A, González A: The effect of verbal instructions on blood pressure measurement. J Hypertens 1993; 11: 293-296.

32. Williams RB Jr, Kimball CP, Williard HN: The influence of interpersonal interaction on diastolic blood pressure. Psychosom Med 1972; 34: 194-198.

33. Imai $Y$, Satoh $\mathrm{H}$, Nagai $\mathrm{K}$, et al: Characteristics of a community-based distribution of home blood pressure in Ohasama in northern Japan. J Hypertens 1993; 11: 1441-1449.

34. Julius S, Ellis CN, Pascual AV, et al: Home blood pressure determination: value in borderline ("labile") hypertension. JAMA 1974; 229: 663-666.

35. Bättig B, Steiner A, Jeck T, Vetter W: Blood pressure self-measurement in normotensive and hypertensive patients. J Hypertens 1986; 7 (Suppl 3): S59-S63.

36. Mejia AD, Julius S, Jones KA, Schork NJ, Kneisley $\mathrm{J}$ : The Tecumuseh Blood Pressure Study: normotensive data on blood pressure self-determination. Arch Intern Med 1990; 150: 1209-1213. 